\title{
Influence of Objective Three-Dimensional Measures and Movement Images on Surgeon Treatment Planning for Lip Revision Surgery
}

Carroll-Ann Trotman, B.D.S., M.A., M.S., Associate Dean and Professor, University of Maryland School of Dentistry, Baltimore, MD, and Adjunct Professor, Department of Orthodontics, School of Dentistry, University of North Carolina at Chapel Hill, Chapel Hill, NC

Ceib Phillips, Ph.D.,

Assistant Dean and Professor, School of Dentistry, University of North Carolina at Chapel Hill, Chapel Hill, NC

Julian J. Faraway, Ph.D., Professor, Department of Mathematical Sciences, University of Bath, Bath, England

Terry Hartman, and Clinical Research Assistant, Facial Animation Laboratory, School of Dentistry

John A. van Aalst, M.D., M.A., F.A.C.S., F.A.A.P.

Associate Professor of Surgery, Division of Plastic Surgery, and Adjunct Faculty, Department of Biomedical Engineering, University of North Carolina at Chapel Hill, Chapel Hill, NC

\section{Abstract}

Objective-To determine whether a systematic evaluation of facial soft tissues of patients with cleft lip and palate, using facial video images and objective three-dimensional measurements of movement, change surgeons' treatment plans for lip revision surgery.

Design-Prospective longitudinal study.

Setting-The University of North Carolina School of Dentistry.

Patients, Participants-A group of patients with repaired cleft lip and palate $(\mathrm{n}=21)$, a noncleft control group $(\mathrm{n}=37)$, and surgeons experienced in cleft care.

Interventions-Lip revision.

Main Outcome Measures-(1) facial photographic images; (2) facial video images during animations; (3) objective three-dimensional measurements of upper lip movement based on $Z$

\footnotetext{
(c) Copyright 2013 American Cleft Palate-Craniofacial Association

Address correspondence to: Dr. Carroll-Ann Trotman, Adjunct Professor, Department of Orthodontics, School of Dentistry, University of North Carolina at Chapel Hill, 3160 Old Dental Building, Campus Box 7450, Chapel Hill, NC, 27599-7450. carrollann_trotman@dentistry.unc.edu, ctrotman@umaryland.edu..

Since the study, I am much more aware of movement. I will now have the patients purse their lips, do a cheek puff, smile in wide and low amplitude. I may even ask them to grimace. Though not always, I may also take a picture of the person with these facial expressions. Before the study, I was aware primarily of form, but now am much more conscious of function. I dissect the function into vertical and horizontal movement, rather than just getting an impression of overall movement. Another way to say this is that I am aware of the pieces of movement. I break them apart during the evaluation in order to come up with my overall gestalt. Because I am breaking down movement, I am also much more aware of what scar tissue is doing to the components of movement, and not just to form. Form is clearly important to the aesthetics, yet function is equally important. We are very aware of this [fact] with the palate. We don't see the palate, and can only hear what the palate is doing, by listening to clear (or hypernasal) qualities of the speech. My growing realization about the components of movement of the lip, [I believe] have made me a better surgeon. (J.V.A.)
} 
scores; and (4) objective dynamic and visual three-dimensional measurement of facial soft tissue movement.

Results-With the use of the video images plus objective three-dimensional measures, changes were made to the problem list of the surgical treatment plan for $86 \%$ of the patients $(95 \%$ confidence interval, 0.64 to 0.97 ) and the surgical goals for $71 \%$ of the patients ( $95 \%$ confidence interval, 0.48 to 0.89 ). The surgeon group varied in the percentage of patients for whom the problem list was modified, ranging from $24 \%$ (95\% confidence interval, $8 \%$ to $47 \%$ ) to $48 \%(95 \%$ confidence interval, $26 \%$ to $70 \%$ ) of patients, and the percentage for whom the surgical goals were modified, ranging from $14 \%$ (94\% confidence interval, $3 \%$ to $36 \%$ ) to $48 \%$ (95\% confidence interval, $26 \%$ to $70 \%$ ) of patients.

Conclusions-For all surgeons, the additional assessment components of the systematic valuation resulted in a change in clinical decision making for some patients.

\section{Keywords}

dynamics; lip revision; soft tissue; treatment planning

An infant born with a cleft of the lip and palate (CLP) will undergo primary surgical repair of the lip soon after birth to correct the cleft defect. With age, however, residual disfigurement and impairment in the lips may become more apparent due to the effects of scarring. Additional lip revision surgery later in life may be recommended to improve lip function and aesthetics. The age at which this surgery is performed tends to follow a bimodal distribution - at around 4 to 8 years and also during the middle to late teenage years (Marsh, 1990). The decision to perform revision surgery is made by the surgeon in conjunction with the parents and/or patient, and is based on a wide range of clinical indicators (Marsh, 1990; Henkel et al., 1998). A few examples of indicators for lip revision from one craniofacial center included whistle-type deformity, notching of the upper lip, conspicuous scarring, disturbance of the lip red/white border or the upper/lower lip relation, and unusual lip segments (Henkel et al., 1998). Clinically, the repaired cleft not only affects facial aesthetics but also function. In the past, functional components/impairments in movement generally were not considered, given the current standard-of-care reliance on mainly static evaluations. Even when surgeons do assess the function, they have no quantitative and visual aids to incorporate functional assessment into treatment planning and decisions regarding surgical goals.

There have been only a few studies in which professionals rated nasolabial movements (Morrant et al., 1996; Ritter et al., 2002; Trotman et al., 2003; Trotman et al., 2007). The results demonstrated considerable bias and poor interrater reliability among the raters, a finding that led Morrant and Shaw (1996) to conclude that research in this area was sorely lacking and other researchers to recommend the development of more objective measures of facial movement (Salgado et al., 2010). Our own findings in this regard indicated poor agreement among surgeons experienced in cleft care when rating the severity of the cleft scar in patients whose faces were in repose and during various animated movements (Trotman et al., 2007). Specifically, surgeons' consistency in rating repeated facial views of the same patient was moderate to excellent; however, the overall agreement among surgeons when rating individual patients was low to moderate. Moreover, the surgeons demonstrated wide variation in their recommendations for (or against) "the need for revision surgery" when evaluating these same patients (Trotman et al., 2007). These conflicting and often confusing findings attest to the need for more patient-centered, objective measures to supplement surgeons' subjective evaluation, along with a standardized methodological approach for evaluation that can be used for treatment planning and to assess treatment outcomes (Salgado et al., 2010; Trotman et al., 2010). 
Over the past several years, our research team has developed and demonstrated the utility of novel objective techniques to improve diagnosis, treatment planning, and outcomes for lip repair and revision (Trotman et al., 2007; Tanikawa et al., 2010; Trotman et al., 2010; Faraway et al., 2011; Trotman, 2011). These research developments have been part of an ongoing clinical trial (NCT00070811). One aim of this trial was to determine the impact of a "systematic" and standardized approach for the diagnosis and treatment planning of lip revision surgery in patients with CLP that combines a subjective static and dynamic evaluation with the use of objective three-dimensional (3D) measures of patients' soft tissue movements. The 3D measures were based on (1) $z$ scores that represented a single measurement of the difference in a patient's impaired movement from that of averaged normal movement, and (2) an objective dynamic 3D measurement (ODM) based on a 3D visual, dynamic, and statistical comparative modeling of a patient's mean facial movements against mean control movements for a particular facial animation.

Accordingly, the specific aims of this study were as follows. (1) In the case of the operating surgeon who performed the lip revision surgery, to determine whether a systematic evaluation approach that combines objective $z$ score measurements of facial movement and facial video images, when added to the surgeon's original treatment plan, changed the surgeon's plan and goals for lip revision in patients with CLP. (2) In the case of a group of surgeons experienced in cleft care, to determine whether a systematic evaluation approach that combines the ODM and facial video images, when added to surgeons' evaluation of still photographs, changes the plan and goals for lip revision surgery in patients with CLP. The hypothesis was that the use of the systematic evaluation approach changes surgeons' original treatment plan and goals for lip revision surgery.

\section{Method}

This prospective, longitudinal study was part of a larger clinical trial (NCT00070811) designed to evaluate the functional outcomes of cleft lip revision surgery (Trotman et al., 2007; Trotman et al., 2010). The control group for the clinical trial provided baseline normative data on facial movements during different animations; whereas, the CLP group comprised a wide range of patients with facial disfigurement and impaired movements. For this study, the sample consisted of a group of 21 children with repaired CLP recruited from the University of North Carolina (UNC) Craniofacial Center and a group of 37 noncleft control children recruited from the Orthodontic and Pediatric Dentistry clinics at UNC. The children with CLP were evaluated for lip revision surgery by a single surgeon (J.V.A.) at an initial clinical examination. For the children who met the study selection criteria (Table 1), the research assistant contacted the parents to explain the purpose and protocol of the study. Informed consent/assent was obtained and the child was enrolled. The consent and Health Insurance Portability and Accountability Act documents were approved by the UNC Biomedical Human Subjects Institutional Review Board, and no child was excluded from participation on the basis of sex or race.

The sample of children with CLP represented consecutive patients, who along with their parent(s) and/or guardian(s) consented to participate in the study. The gender and mean ages for the CLP patients and noncleft subjects are provided in Table 2. Of the 21 patients, 19 had a CLP and two had a cleft lip only. Of the 19 with a CLP, five were bilateral; and of the two patients with cleft lip only, one was bilateral. There were 14 white Americans, six Hispanic Americans, and one Pacific Islander. There were twice as many boys as girls, and on average, the boys were older than the girls by approximately 5 years (Table 2). Of the 37 noncleft subjects, there were 30 white Americans, five African Americans, one Hispanic American, and one Asian American. Also, there were slightly fewer girls in this group, and the boys were older than the girls on average by 2 years. As part of the original clinical trial, 
each child with a CLP was seen for four test visits/sessions: two sessions before lip revision surgery at 1 to 3 months before and just before the surgery; and two sessions after the surgery at 3 and 12 months (Trotman et al., 2007). Similar data on the noncleft children were collected at these same time points. At each visit, data were collected to document and assess problems associated with the patients' static facial form, dynamic facial form, and 3D dynamic facial movement when modeled statistically. Because this study evaluated treatment planning for lip revision, only the data from the two presurgery visits were used.

\section{Data for Subjective Assessment of Form}

Static and dynamic (animated) facial form data were collected for subjective assessment by the surgeons. Facial form was assessed from a standardized series of frontal, right and left profile, and worm's-eye facial photographic views of each patient taken at rest and smiling (Fig. 1) and from a series of video images of facial animations or movements: smile, natural smile, cheek puff, lip purse, mouth opening, and grimace (Fig. 2). These presurgery views then were assembled on a DVD for evaluation by the surgeon.

\section{Data for Objective Measurement of Movement}

A video-based tracking system (Motion Analysis, Motion Analysis Corporation, Santa Rosa, CA) was used to assess facial movement according to the method of Trotman and colleagues (Fig. 3) (Trotman et al., 2007). The system tracked the 3D movement of retroreflective markers attached to specific facial landmarks. This approach for objective evaluation has been published extensively. Here, a brief summary of the approach is provided (Trotman et al., 1998; Trotman et al., 2000). The system first was calibrated. The capture volume in which the patient's head was located was calibrated using a frame in the shape of an L. Four analog video cameras with a $25-\mathrm{mm}$ focal length sampled the positions of the markers at 60 $\mathrm{Hz}$. The $\mathrm{L}$ frame had four markers fixed to exact and known locations on the frame. Next, the research assistant waved a wand with precisely located markers throughout the capture volume. This process located the exact camera positions, accounted for any geometric distortions in the camera lenses, and accurately measured the camera lens focal lengths. The error in specifying marker position within the system was determined previously and approximated $0.53 \mathrm{~mm}$ (standard deviation $=0.45 \mathrm{~mm}$ ) (Trotman et al., 1998; Trotman et al., 2000). Then, the child was positioned within the calibrated area and instructed to perform five maximal facial animations from rest: smile, cheek puff, lip purse, mouth opening, and grimace. A natural smile movement also was elicited in response to the operator's smile. Prior to each animation, the research assistant mimed the animation. Practice trials were given, after which 10 trials of each facial animation were recorded. Images from the cameras were digitized in real time for subsequent off-line processing.

Objective 3D data were processed for five of the six animations-smile, natural smile, cheek puff, lip purse, and mouth opening. The grimace animation was not included. For each replication (10) of each animation (five) for each of the facial landmark markers (38) on the face, a time series of 3D vectors were obtained defined by $(x, y, z)$ where $x, y$, and $z$ represented the position in space recorded at 1/60-second intervals for 4 seconds. Then for each presurgery visit and animation, the overall, horizontal, and vertical upper lip movements were computed for each patient as well as a measurement of asymmetry.

Overall Measurement-This measurement was based on the change over time from rest to the completion of the animation in distances between eight landmarks on the upper lip (Fig. 4a). Let $d_{i j}(t)$ be the distance between landmarks $i$ and $j$ at time $t$. Then, let $r_{i j}(t)\left(d_{i j}(t) /\right.$ $\left(\mathrm{d}_{\mathrm{ij}}(0)\right)-1$ represent the relative change in the distance from rest for a particular animation. The max $\left|r_{i j}(t)\right|$, which represented the maximum absolute relative change from rest, was computed. The eight landmarks resulted in 28 pairwise distances between these landmarks, 
and the summary measurement for each animation was the average (u) of the logged values over all replicates of the landmark pairs for a particular animation where

$$
u=1 / 28 \Sigma \log \max \left|r_{i j}(t)\right| \text {. }
$$

Horizontal Measurement-This measurement was based on the change over time from rest to the completion of the animation in distance between all pairs among landmarks 2 to 5 (six pairs) and then again, all pairs among landmarks 7 and 8 (six pairs) on the upper lip, giving a total of 12 pairs (Fig. 4b). The average horizontal measurement of movement then was calculated similar to the overall measurement.

Vertical Measurement-This measurement was based on the change over time from rest to the completion of the animation in distance between the four vertical pairs of landmarks and the diagonal pairs on the upper lip, giving a total of 10 pairs (Fig. 4c). The average vertical measurement of movement then was calculated similar to the overall measurement.

Asymmetry Measurement-The asymmetry of movement was calculated as follows. First, separate averages of the maximum relative change over time from rest to the completion of the animation were computed for pairwise distances on the right and left sides of the nasolabial region. Then, the absolute difference between the right and left sides was computed to give a measure of the extent of the asymmetry.

Then, an animation/visit/patient-specific $z$ score was calculated from the mean measurements for each patient/visit/animation and the respective means and standard deviations from the first and second visits of the noncleft control subjects. For each animation, the $z$ scores represented one objective measurement of the magnitude of difference between a patient's average movement relative to the control group's average movement in standardized deviations. For all the measurements, the greater the magnitude of the $z$ score, the further away the movement was from the control movement for a particular patient. For the overall, vertical, and horizontal measurements, a negative $z$ score implied a decrease in the movement and a positive $z$ score represented an increase. The measurement of asymmetry was always positive, because it represented the absolute difference in movement between the right and left sides of the nasolabial region. Thus, the greater the difference in this positive value, the greater was the asymmetry.

\section{Operating Surgeon's Clinical Exam/Diagnosis/Systematic Evaluation (Specific Aim 1)}

For a patient attending the UNC Craniofacial Center for lip revision surgery, the surgeon (J.V.A.) performed the following for each patient.

1. An initial clinical evaluation (baseline). Depending on the patient's age, the patient's face was observed at rest and during animated behaviors of talking, smiling, and laughing. During the "at rest" evaluation, the face was assessed for the presence or absence of asymmetry of the nose and lips; and for abnormalities in the vertical, horizontal, and anterior-posterior facial dimensions. The rigidity of the nasolabial tissues also was assessed by lightly touching areas around the patient's lip and nose to determine the extent of scarring. On occasion, the surgeon might record his own "static" facial photographs of the patient.

2. Subjective evaluation of the series of facial photographic views.

3. Subjective evaluation of the animated facial video images plus the use of the $Z$ score objective measurement of movement for each animation. 
At the end of evaluation stages 1,2, and 3, the surgeon provided a detailed problem list and a list of desired goals that would be achieved as a result of the revision surgery. The main goals for stages 2 and 3 were any of the following: increase or decrease the vertical movement of upper lip; increase or decrease the lateral movement of upper lip; improve the symmetry of upper lip movement; and no change in upper lip movement indicated (change the lip form only).

\section{Surgeon Group Systematic Evaluation (Specific Aim 2)}

Four surgeons experienced in cleft treatment viewed the images of the patients. Each surgeon performed a systematic evaluation of each patient and provided a detailed problem list and desired goals. Unlike the operating surgeon (J.V.A.), the four surgeons had no direct patient contact, and therefore, they did not conduct an initial clinical evaluation. They viewed the patients' images on the DVD only. Thus, the sequence of the evaluation was somewhat abbreviated as follows. (1) Subjective evaluation of a series of facial photographic views to assess static facial form. (2) Subjective evaluation of a series of animated facial video images to assess dynamic facial form plus viewing the ODM for each animation.

The $z$ score measurement was replaced with the more comprehensive ODM statistical and visual comparison of each patient's averaged facial movement with the averaged control facial movement (Fig. 5). The statistical method underlying this modeling has been published elsewhere (Trotman et al., 2010; Faraway et al., 2011; Trotman, 2011). Figure 5 shows composite views of the face and corresponding facial landmarks for patient (P) 21 during smile, lip purse, and cheek puff animations. The video frames and ODM are frozen at the maximum of the movement for each animation. The patient's landmarks are solid circles and the control landmarks are open black circles. A dynamic demonstration of this approach can be seen at http://people.bath.ac.uk/jjf23/face/planning/ for several patients (P11, P12, and P21). First, the individual repetitions of the movement by each patient are shown, followed by a comparison of the individual repetitions of the patient's movements superimposed on the mean normal or control movement for each animation, and finally a comparison of the patient's mean movements superimposed on the normal or control mean movement. During this dynamic comparison, the patient's landmarks were solid red circles and the control landmarks were open black circles. This statistical modeling was viewed by the surgeon group.

\section{Statistical Analyses}

The primary outcome measure for this study was the extent to which the animated dynamic video images in conjunction with the objective 3D measures of movement-either the $Z$ scores used by the operating surgeon or the ODM comparisons used by the surgeon groupchanged the original targeted goals and problem list of the operating surgeon. This change reflected the influence/utility of the objective measures of movement and the dynamic videos on the decision of the surgeon (Han et al., 1991; Callahan et al., 2005). We hypothesized that the additional information from the animated videos and objective measures would produce a change in the problem list or treatment goal for a majority of patients. Assuming an expected proportion of 0.60 , with a sample size of 24 , a two-sided $95 \%$ confidence interval (CI) for a single proportion using the large sample normal approximation would extend 0.2 from the observed proportion.

On a general level, given the additional information, surgeons may change the desired goals for the treatment plan or the problem list in the following ways: (1) Maintain both the problem list and the surgical goals as previously stated; (2) modify the problem list but maintain the previous goals; (3) maintain the previous problem list but modify the previous 
goals; or (4) modify both the problem list and the surgical goals. A modification was counted as a change by the surgeon and coded as yes for that patient. If no modification was made, the decision was counted as no change and coded as no. At each sequential evaluation at which the additional information was provided to the single surgeon (e.g., after the single surgeon's combined clinical evaluation and evaluation of the still photographs, and then after the surgeon's combined clinical evaluation, evaluation of still photographs, and evaluation of dynamic video with the objective $z$ score measurement), and to each member of the surgeon group (e.g., after each surgeon's evaluation of the still photographs, and then after each surgeon's combined evaluation of the video images with the objective ODM comparisons), the proportion of yes responses indicating a change in the problem list or goal for surgery was calculated.

\section{Results}

\section{Results for the Operating Surgeon}

Each patient's animation $z$ scores at the two presurgery visits for the overall, horizontal, vertical, and asymmetry measurements are shown in Figure 6. The greater the magnitude of the $z$ score for a patient's specific animation, then the greater was the deviation of movement from normal and the greater the impairment in movement. It can be seen that, in general, the pattern of variation of the scores was similar at both presurgery visits, and the greatest scores were seen for the maximum smile, lip purse, and cheek puff animations. These scores were negative, indicating a decrease in lip movement compared with the controls. There also was considerable variation in the scores among the 21 patients in a given visit, and this variation ranged from negative scores of less than 1.0 to negative scores greater than 6 . For example, P12, who had a repaired bilateral CLP had negative scores of magnitude 4 and greater for the maximum smile, lip purse, and cheek puff animations, and P11, who had a repaired left unilateral cleft lip, had negative scores of magnitude less than 3 for all the animations except for lip purse, which had a negative score of magnitude greater than 6. The impairment for P11 involved both the vertical and horizontal components of movement. For some patients the scores changed by as much as 1.5 to 2.0 between the first and second visits, with an overall tendency for the scores to be less in magnitude at the second presurgery visit. Finally, the asymmetry scores did not appear to be substantially different between patients with a unilateral CLP (Fig. 5-P1 to P3, P5 to P11, P14 to P16, P18, and P20) versus a bilateral CLP (Fig. 5-P4, P12, P13, P17, P19, and P21).

The results for the systematic assessment approach by the operating surgeon with the video images plus the $z$ scores were that the surgeon modified the problem list for $33 \%$ of the patients and the surgical goals for $38 \%$ of the patients when the still photographs were provided (Table 3). Modifications were made to the problem list and the surgical goals for substantially more patients when the additional information from the dynamic video plus the $z$ score measurement was provided. As seen in Table 3, the problem list was modified for $86 \%$ of the patients (95\% CI, $64 \%$ to $97 \%$ ) and the surgical goals were modified for $71 \%$ of the patients (95\% CI, $48 \%$ to $89 \%$ ) with the use of the video images plus $z$ scores.

\section{Results for the Surgeon Group}

For the assessment by the four surgeons, the baseline assessment was made from the static or still photographs. As seen in Table 4, modifications were made by all the surgeons to the problem list and goals when the video images and ODM comparisons were added to the evaluation. The surgeons varied in the percentage of patients for whom the problem list was modified, ranging from $24 \%$ (95\% CI, $8 \%$ to $47 \%$ ) to $48 \%$ (95\% CI, $26 \%$ to $70 \%$ ) of patients, and the percentage for whom the surgical goals were modified, ranging from $14 \%$ (94\% CI, 3\% to $36 \%$ ) to $48 \%$ (95\% CI, $26 \%$ to $70 \%$ ) of patients. 


\section{DISCUSSION}

In this study, a systematic approach for surgical treatment planning was described. The approach, which is designed to be used by surgeons, uses facial photographs, videos of the animated face, and 3D objective measures. The findings demonstrated that the use of videos of patients' faces combined with objective 3D measures altered the surgeon's treatment plan for a significant number of patients. One could infer that this additional information was useful for the surgeon's treatment planning process. Surgeons develop an overall gestalt of a patient's facial disability that is based on a variety of clinical indicators combined with their own individual clinical experience. As might be expected, surgical experience varies, and the specific patient factors considered by different surgeons during treatment planning also would be variable. Thus, it is not surprising that numerous rating schemes to evaluate treatment have been proposed with inconclusive and contradictory results (Eliason et al., 1991; Tobiasen et al., 1991; Lo et al., 2002; Papamanou et al., 2009). The advantage of the evaluation approach presented here is that it preserves an individual surgeon's need to gain his or her overall impression of the patient's facial disability but systematizes the diagnostic process by adding movement videos and supplements the diagnosis with objective measurements. Moreover, as with any innovative technique, there is an expectation that a learning curve will exist regarding how the new information would be incorporated into surgical approaches and goals. This learning curve may have been the reason that J.V.A., who was the operating surgeon involved in the evolution of the systematic approach, modified the problem list and surgical goals for a substantially higher proportion of subjects when compared with the group of surgeons. These four surgeons, although they were trained in the use of the approach/technique, were not accustomed to the incorporation of these novel data.

The objective $z$ score measurement used in this study varied greatly among the patients, a fact attesting to the individualized nature of the movement impairment in patients with CLP that results from both the cleft lip defect and the initial cleft lip repair. Also, based on the greater $z$ scores for the maximum smile, lip purse, and cheek puff animations, it appears that these animations were most affected by the cleft defect and subsequent surgical repairs. The scores at the second presurgery patient visit tended to be slightly smaller than those at the first visit, and this finding probably represented a learning effect on the part of the patients and the noncleft controls. Moreover, the difference between the scores at the two visits varied by as much as two standard deviations, indicating that a $z$ score less than 3 probably does not represent sufficient impairment to be of concern. However, because the $z$ scores represented a univariate measurement of the difference in impairment between the patients and the controls, our research group has since developed a multivariate approach to measure this difference and capture the full nature of the movement differences (Trotman et al., 2010; Faraway et al., 2011). The approach is the dynamic statistical modeling of the differences (ODM), which is much more robust. Surgeons can more accurately determine areas of the face that are impaired by visually comparing the mean movement of a patient's facial landmarks for an animation superimposed on that of the mean movement of the control-group landmark movement.

This study had several additional aspects, some of which the authors acknowledge as limitations, that warrant further discussion. (1) Given this approach, the acquisition of a greater library of control subject data is necessary so that patients with CLP can be compared with age- and gender-matched controls. (2) The approach used was shown to provide information that the surgeon uses. It remains to be seen whether surgeons can use the approach to improve patient outcomes and to reach consensus on their decisions. Our research team will continue to investigate this aspect. (3) This study assessed whether the use of the videos and the objective measures altered the surgeon's original treatment plan. It 
did not assess what the surgeon (J.V.A.) did during surgery to ensure that he achieved his goals. That aspect of this research is highly detailed and is the subject of future research. (4) This approach for the evaluation of facial movements does require training of the surgeon in the use of the assessment. To accomplish this training photographic, video, and "objective" data of previous patients, both in terms of $z$ scores and the ODM, have been assembled on DVD to be viewed and analyzed by surgeons. (5) It is possible that the statistical analysis of the impact of this approach on the surgeon's treatment planning decisions underestimated the effect of a change. This could have occurred if the surgeon maintained his original treatment plan because he felt the additional information confirmed his initial decisions. This possibility was not measured. (6) The learning effect for the operating surgeon (Specific Aim 1) discussed earlier is a strong possibility. This surgeon, either knowingly or unknowingly over time, may have incorporated the information presented in the videos into his initial treatment planning decisions by being more observant of the patients' animated behaviors during his initial clinical examinations. This fact would seem likely given the surgeon's own description of the value of the movement videos.

\section{Acknowledgments}

This study was supported by grants R01 DE13814-01A1 from the National Institute of Dental and Craniofacial Research (NIDCR) to Dr. Carroll-Ann Trotman.

\section{REFERENCES}

Callahan C, Sadowsky LP, Ferreira A. Diagnostic value of plaster models in contemporary orthodontics. Semin Orthod. 2005; 11:94-97.

Eliason MJ, Hardin MA, Olin WH. Factors that influence ratings of facial appearance for children with cleft lip and palate. Cleft Palate Craniofac J. 1991; 28:190-194. [PubMed: 2069976]

Faraway JJ, Trotman C-A. Shape change along geodesics with application to cleft lip surgery. J R Stat Soc Ser C Appl Statist. 2011; 60:743-755.

Frans AF, van Zuijlen PPM, Don Griot JPW, van der Horst CMAM. Assessment of scar quality after cleft lip closure. Cleft Palate Craniofac J. 2012; 49:171-176. [PubMed: 21851284]

Han UK, Vig KWL, Weintraub JA, Vig PS, Kowalski CJ. Consistency of orthodontic treatment decisions relative to diagnostic records. Am J Orthod Dentofac Orthop. 1991; 100:212-219.

Henkel K-O, Gundlach KKH, Saka B. Incidence of secondary lip surgeries as a function of cleft type and severity: one center's experience. Cleft Palate Craniofac J. 1998; 35:310-312. [PubMed: 9684768]

Lo L-J, Wong F-H, Mardini S, Chen Y-R, Noordhoff MS. Assessment of bilateral cleft lip nose deformity: a comparison of results as judged by cleft surgeons and laypersons. Plast Reconstr Surg. 2002; 110:733-738. [PubMed: 12172130]

Marsh J. When is enough enough? Secondary surgery for cleft lip and palate patients. Clin Plast Surg. 1990; 17(1):37-47. [PubMed: 2302918]

Morrant DG, Shaw WC. Use of standardized video recordings to assess cleft surgery outcome. Cleft Palate Craniofac J. 1996; 33(2):134-142. [PubMed: 8695621]

Papamanou DA, Gkantidis N, Topouzelis N, Christou P. Appreciation of cleft lip and palate treatment outcome by professionals and laypeople [published online ahead of print Month Day, 2009]. Eur J Orthod.

Ritter K, Trotman C-A, Phillips C. Validity of subjective evaluations for the assessment of lip scarring and impairment. Cleft Palate Craniofac J. 2002; 39:587-596. [PubMed: 12401105]

Salgado MD, Curtiss S, Tollefson TT. Evaluating symmetry and facial motion using 3D videography. Facial Plast Surg Clin N Am. 2010; 18:351-356.

Tanikawa C, Takada K, van Aalst J, Trotman C-A. Objective 3D assessment of lip form in patients with repaired cleft lip. Cleft Palate Craniofac J. 2010; 47:611-622. [PubMed: 20500076]

Tobiasen JM, Hiebert JM, Boraz RA. Development of scales of severity of facial impairment. Cleft Palate Craniofac J. 1991; 28:419-424. [PubMed: 1742313] 
Trotman C-A. Faces in 4D-why do we care and why the 4th dimension? Am J Orthod Dentofacial Orthop. 2011; 140:895-899. [PubMed: 22133956]

Trotman C-A, Faraway JJ. Sensitivity of a method for the analysis of facial mobility: II. Interlandmark separation. Cleft Palate Craniofac J. 1998; 35:142-153. [PubMed: 9527311]

Trotman C-A, Faraway JJ, Essick GK. 3-D Nasolabial displacement during movement in repaired cleft lip and palate patients. J Plast Reconstr Surg. 2000; 105:1273-1283.

Trotman C-A, Faraway JJ, Losken HW, van Aalst J. Functional outcomes of cleft lip surgery. Part II: quantification of nasolabial movement. Cleft Palate Craniofac J. 2007; 44:607-616. [PubMed: 18177193]

Trotman C-A, Faraway JJ, Phillips C. Visual and statistical modeling of facial movement in patients with cleft lip. Cleft Palate Craniofac J. 2005; 42:245-254. [PubMed: 15865457]

Trotman C-A, Faraway JJ, Phillips C, van Aalst J. Effects of lip revision surgery in cleft lip/palate patients. J Dent Res. 2010; 89:728-732. [PubMed: 20439935]

Trotman C-A, Faraway JJ, Silvester KT, Greenlee GM, Johnston LE Jr. Sensitivity of a method for the analysis of facial mobility: I. Vector of displacement. Cleft Palate Craniofac J. 1998; 35:132-141. [PubMed: 9527310]

Trotman C-A, Phillips C, Essick GK, Faraway JJ, Barlow SM, Losken HW, van Aalst J, Rogers L. Functional outcomes of cleft lip surgery. Part I: study design and surgeon ratings of lip disability and the need for lip revision. Cleft Palate Craniofac J. 2007; 44:598-606. [PubMed: 18177192]

Trotman C-A, Phillips C, Faraway JJ, Ritter K. Association between subjective and objective measures of lip form and function: an exploratory analysis. Cleft Palate Craniofac J. 2003; 40:241-248. [PubMed: 12733951]

Trotman C-A, Stohler CS, Johnston LE Jr. Measurement of facial soft tissue mobility in man. Cleft Palate Craniofac J. 1998; 35:16-25. [PubMed: 9482219] 


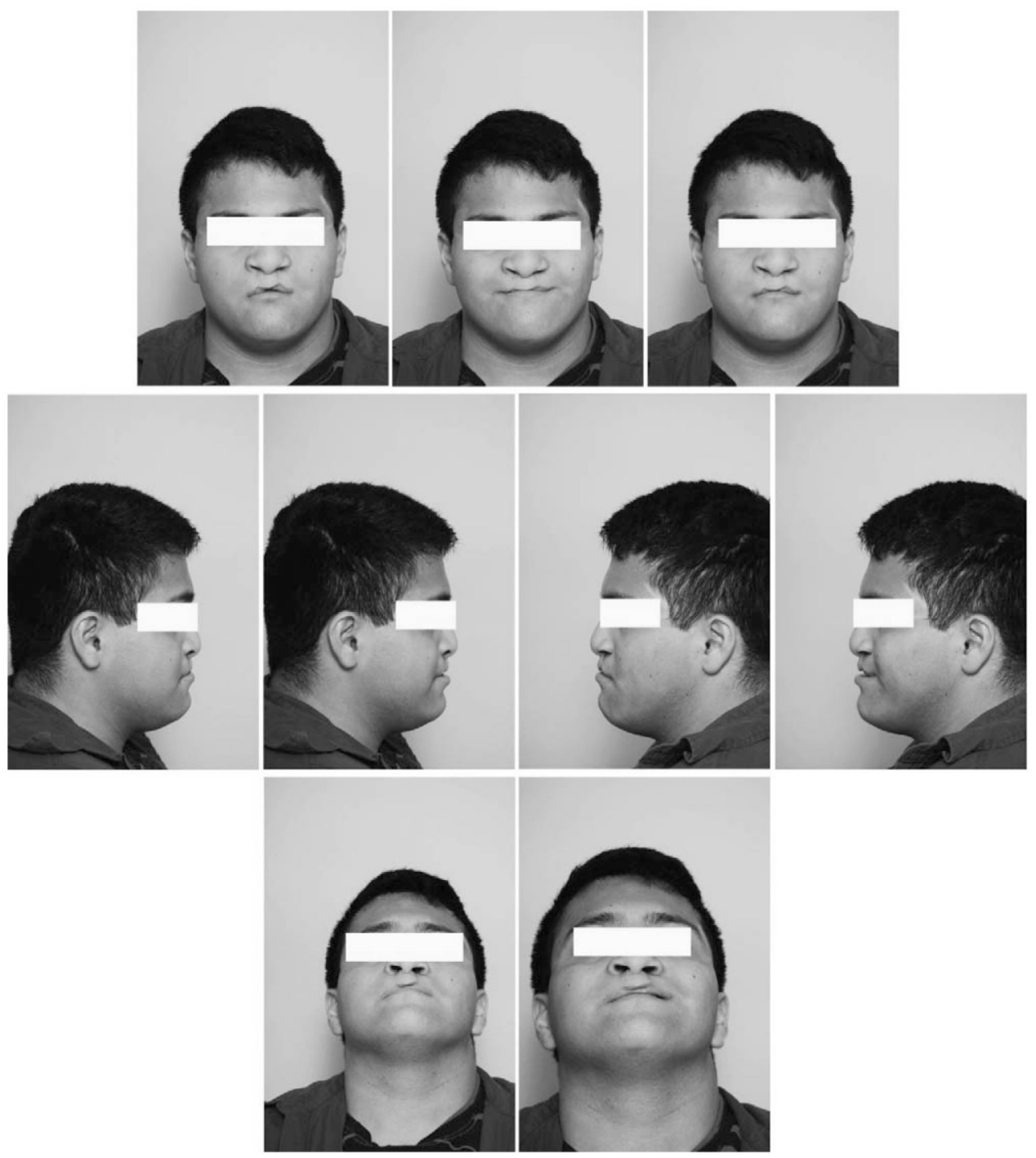

FIGURE 1.

Standardized frontal, right and left profile, and worm's-eye facial photographic views of a patient at rest and while smiling. 


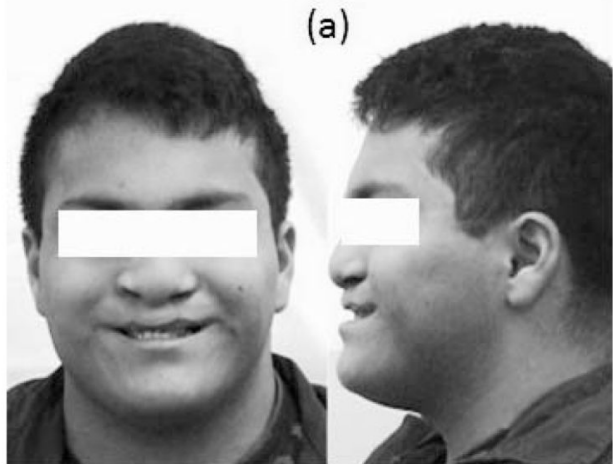

(c)

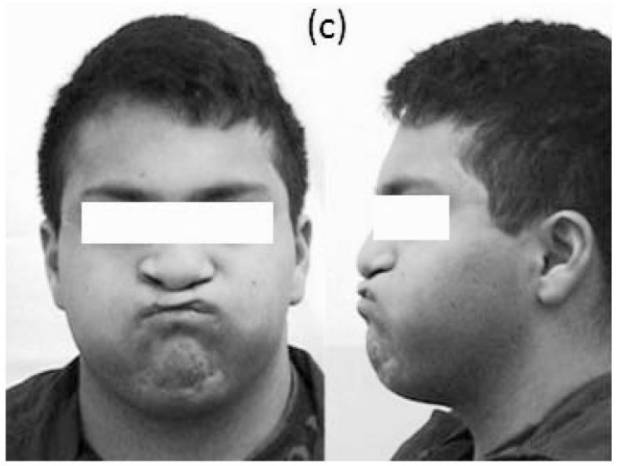

(e)
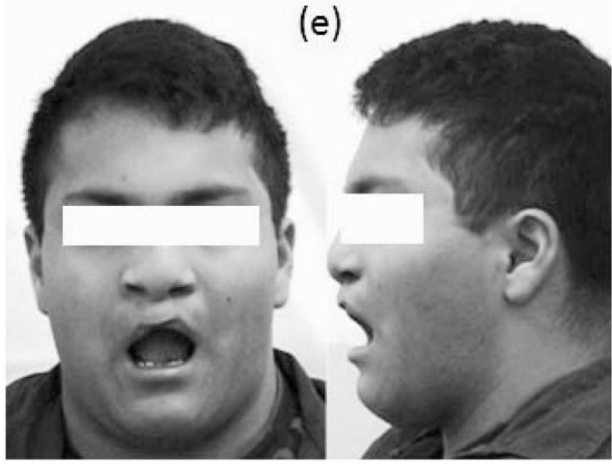

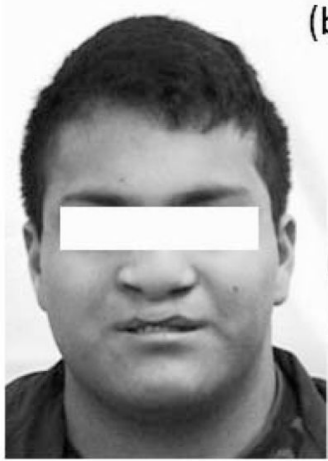

(b)

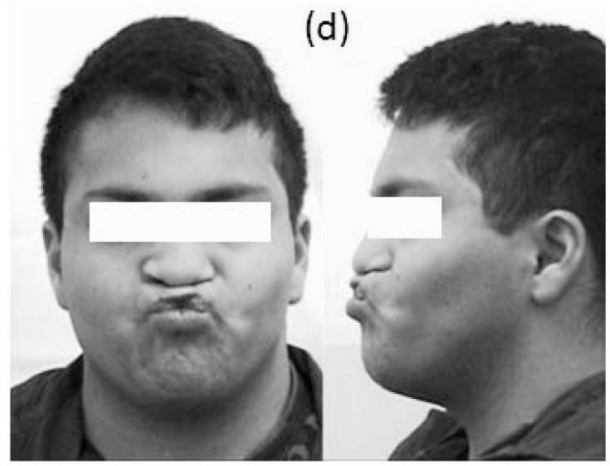

(f)
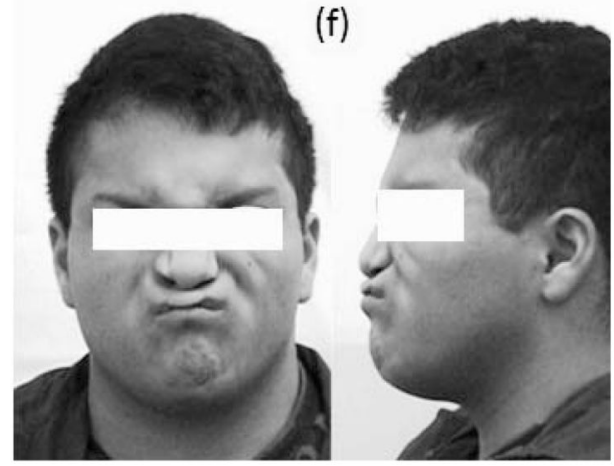

FIGURE 2.

Dynamic video images. a: maximum smile; b: natural smile; c: cheek puff; d: lip purse; e: mouth open; f: grimace. 


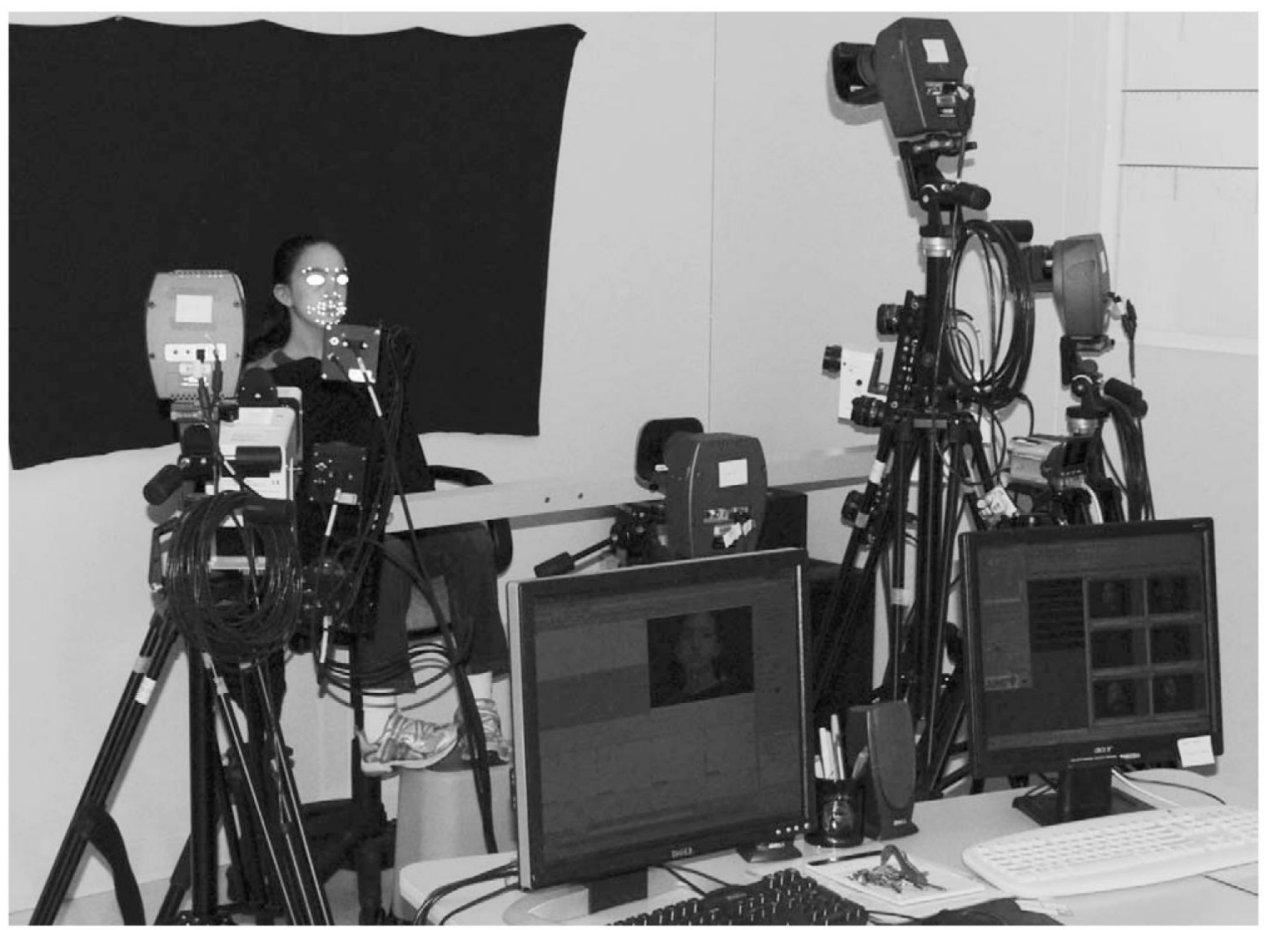

FIGURE 3.

System configuration. 


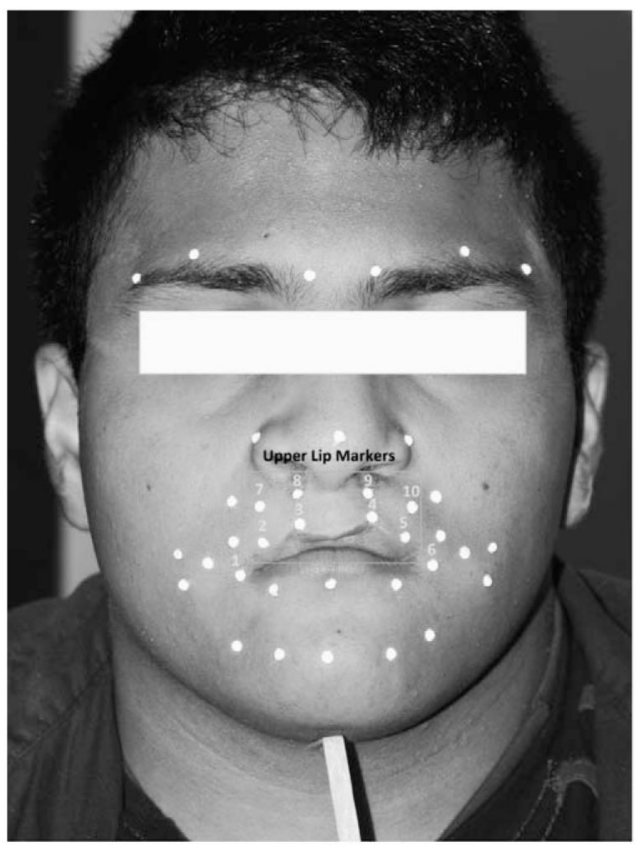

(a)

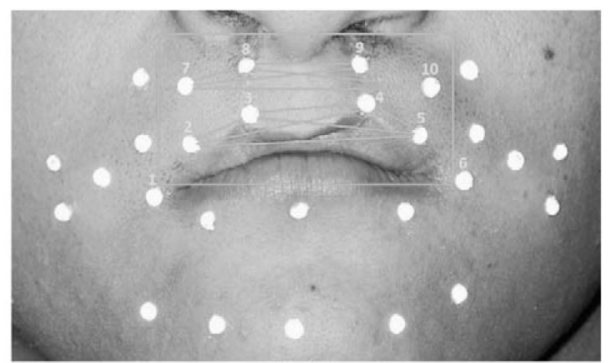

(b)

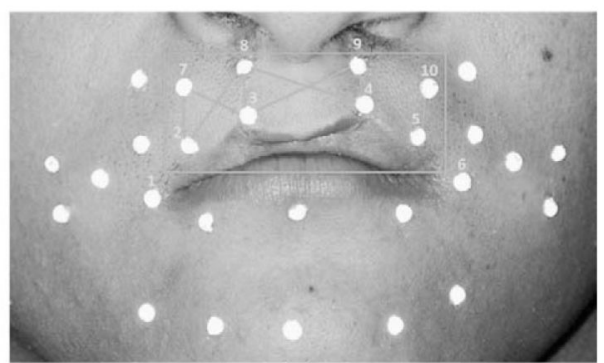

(c)

FIGURE 4.

a: Landmarks for the distortion measurement (7) to (10) and (2) to (5). Definitions: (1) and (6), right and left commissure points located on the right and left commissures, respectively; (3) and (4), right and left upper lip points located on peaks of Cupid's bow; (2), point just above the upper vermillion midway between (1) and (3); (5), point just above the upper vermillion midway between (4) and (6); (7), (8), (9), and (10), points $1 \mathrm{~cm}$ above points (2), (3), (4), and (5), respectively. b: Distances for the horizontal distortion measurement. c: Distances for the vertical distortion measurement. 

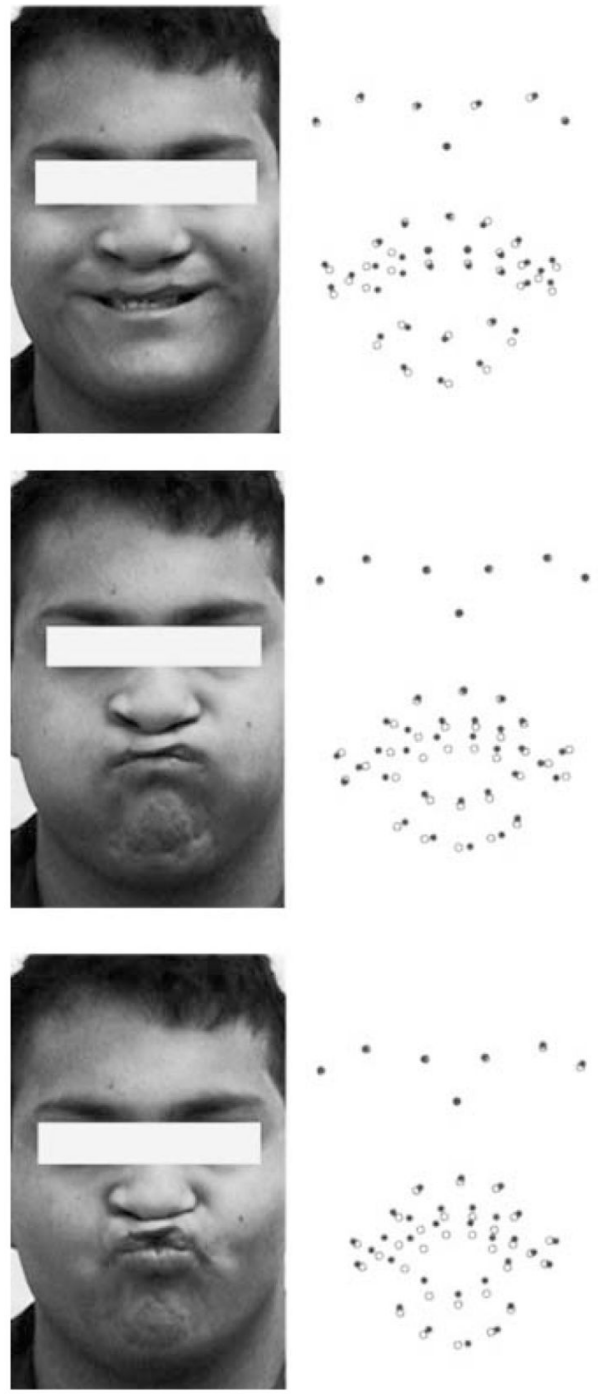

(c)

(a)

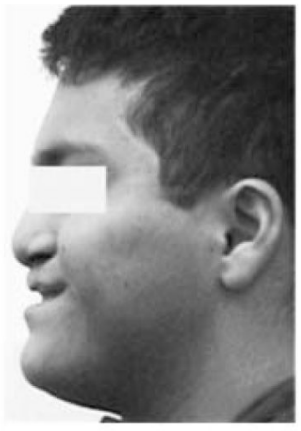

(b)
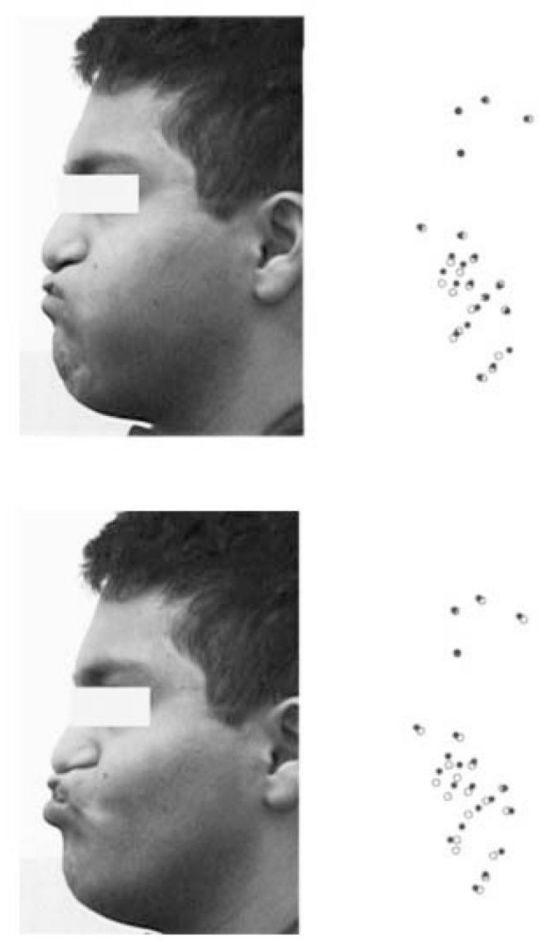

FIGURE 5.

Dynamic video images and corresponding multivariate statistical modeling of animations. The solid gray circles are the patient's movement superimposed on the open black circles that represent the mean movement for the control group. a: maximum smile; b: cheek puff; c: lip purse. 

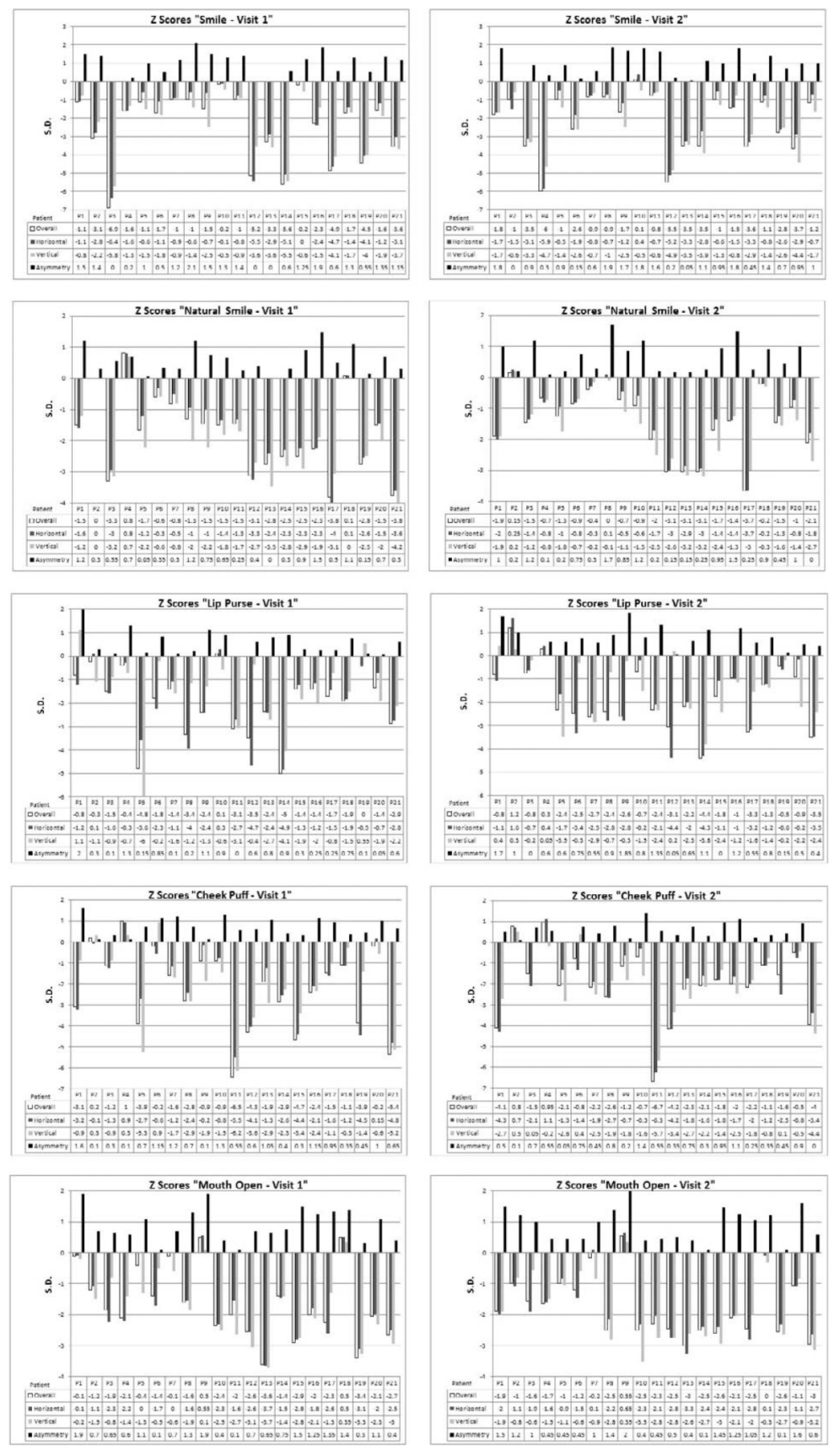

FIGURE 6.

Patients' animation $z$ scores at the two presurgery visits for the overall, horizontal, vertical, and asymmetry measurements. 


\section{TABLE 1}

\section{Study Selection Criteria}

\section{Inclusion Criteria}

- $\quad$ Subject interest and parent willingness to participate in the study.

- An ability to comprehend verbal instructions.

- $\quad$ Specifically for the patients, a previously repaired complete unilateral or bilateral cleft lip with or without a cleft palate

- Age between 5 and 21 years.

\section{Exclusion Criteria}

- Previous orthognathic or facial soft tissue surgery.

- A medical history of diabetes, collagen vascular disease, and/ or systemic neurologic impairment.

- Mental or hearing impairment such that comprehension or ability to perform tests was hampered.

- $\quad$ Specifically for the patients, a lip revision surgery within the preceding 2 years. 


\section{TABLE 2}

\section{Patient Demographics}

\begin{tabular}{lccccc}
\hline & \multicolumn{2}{c}{ Patients with CLP } & & \multicolumn{2}{c}{ Noncleft Control Patients } \\
\cline { 2 - 3 } & Boys & Girls & & Boys & Girls \\
\hline $\mathrm{n}$ & 15 & 6 & 20 & 17 \\
Mean age, y & 15.1 & 9.5 & & 12.5 & 13.0 \\
SD, y & 7.2 & 8.4 & 3.2 & 4.2 \\
\hline
\end{tabular}


TABLE 3

Changes Due to Cumulative Assessments for the Operating Surgeon

\begin{tabular}{lcccc}
\hline \multicolumn{1}{c}{ Assessment Information } & Yes, $\boldsymbol{n} *$ & Proportion Yes & 95\% Exact CI & P Value \\
\hline Clinical (baseline) &. &.. &.. &.. \\
Clinical vs. stills (problems) & 7 & 0.33 & $(0.15-0.57)$ & .10 \\
Clinical vs. stills (goals) & 8 & 0.38 & $(0.18-0.62)$ & .19 \\
Clinical + stills vs. videos + objective measure (problems) ${ }^{\dagger}$ & 18 & 0.86 & $(0.64-0.97)$ & $<.001$ \\
Clinical + stills vs. videos + objective measure (goals) ${ }^{\dagger}$ & 15 & 0.71 & $(0.48-0.89)$ & .04 \\
\hline$* \quad$ & & & &
\end{tabular}


TABLE 4

Changes Due to Cumulative Assessments by the Surgeon Group

\begin{tabular}{lrccc}
\hline \multicolumn{1}{c}{ Assessment Information } & Yes, $\boldsymbol{n}^{*}$ & Proportion Yes & 95\% Exact CI & P Value \\
\hline Stills (baseline) & 7 & 0.33 & $(0.15-0.57)$ & .10 \\
Stills vs. videos + objective measure (problems) ${ }^{\dagger}$ & 18 & 0.86 & $(0.64-0.97)$ & $<.001$ \\
Stills vs. videos + objective measure (goals) ${ }^{\dagger}$ & 15 & 0.71 & $(0.48-0.89)$ & .04 \\
\hline
\end{tabular}

${ }^{\dagger}$ Objective measure $=$ ODM. 